\title{
End Expiratory Pause
}

National Cancer Institute

\section{Source}

National Cancer Institute. End Expiratory Pause. NCI Thesaurus. Code C120929.

The period of time between the end of exhalation and the active start of inspiration. 